\title{
点手性调控的三股铕螺旋体的非对映选择性自组装及圆偏振发光
}

\author{
宋龙飞周妍奸高婷间鹏飞* 李洪峰* \\ (黑龙江大学 化学化工与材料学院 功能无机材料化学教育部重点实验室 哈尔滨 150080)
}

\begin{abstract}
摘要 具有超分子手性的稀土螺旋体为合成高性能稀土圆偏振发光(CPL)材料提供了结构基础. 然而, 稀土 Ln(III)离子 较大的半径和不稳定的配位几何构型为合成高光学纯度的稀土螺旋体带来了困难和挑战. 本工作通过在双三齿配体端 基引入点手性的方式成功构筑了一对儿对映体纯的手性双核三股铕螺旋体, [ $\left.\operatorname{Eu}_{2}\left(\mathbf{L}^{R R}\right)_{3}\right](\mathrm{OTf})_{6}$ 和 $\left[\mathrm{Eu}_{2}\left(\mathbf{L}^{S S}\right)_{3}\right](\mathrm{OTf})_{6}$. 结合 全面的光谱表征和半经验分子力学模型证实了配体端基的点手性成功诱导两个稀土 $\mathrm{Eu}^{3^{+}}$离子采取相同的 $\Lambda$ 或 $\Delta$ 构型, 并形成具有单一 $M$ 或 $P$ 螺旋构象的螺旋体. 配合物的镜像圆二色 $(\mathrm{CD})$ 和 CPL 光谱也进一步验证了一对儿光学纯对映 体的形成. 手性光学性质研究显示螺旋体在乙腈中表现出适中的发光不对称因子 $(|g| u m \mid=0.083)$ 和良好的发光量子产率 $(\mathrm{QY}=19 \%)$. 该方法为制备性能优异的手性稀土 CPL 材料提供了一种可行性参考.
\end{abstract}

关键词 稀土配合物; 手性螺旋体; 手性诱导; 圆偏振发光(CPL)

\section{Point Chirality Regulated Diastereoselective Self-Assembly and Circularly Polarized Luminescence in Eu(III) Triple-Stranded Helicates}

\author{
Longfei Song Yanyan Zhou Ting Gao Pengfei Yan* Hongfeng Li* \\ (Key Laboratory of Functional Inorganic Material Chemistry, Ministry of Education; School of Chemistry, \\ Chemical Engineering and Materials, Heilongjiang University, Harbin 150080, China)
}

\begin{abstract}
The supramolecular chirality of lanthanide helicate offers a structural base to synthesize the excellent lanthanide circularly polarized luminescent (CPL) materials. However, the larger radii and the labile coordination geometries of the $\mathrm{Ln}$ (III) ions make it difficult to control the diastereoselectivity of lanthanide helicates in the self-assembly process. Herein, a pair of chiral dinuclear europium triple-stranded helicates $\left[\operatorname{Eu}_{2}\left(\mathbf{L}^{R R}\right)_{3}\right](\mathrm{OTf})_{6}$ and $\left[\mathrm{Eu}_{2}\left(\mathbf{L}^{S S}\right)_{3}\right](\mathrm{OTf})_{6} \quad\left(\mathbf{L}^{R R / S S}=\right.$ $N, N^{\prime}$-(ethane-4,4'-diyl)bis[6-(R/S)-(1-phenethylcarbamoyl)-pyridine-2-dimethylamide]) were synthesized via point chirality induced strategy. The ligands $\mathbf{L}^{R R / S S}$ were composed of two chiral 2,6-dipicolinic amides as coordination units and ethylenediamine moieties as spacers. General procedure for the preparation of ligands $\mathbf{L}^{R R / S S}$ and corresponding europium complexes $\left[\mathrm{Eu}_{2}\left(\mathbf{L}^{R R / S S}\right)_{3}\right](\mathrm{OTf})_{6}:$ 6-(Methoxycarbonyl)picolinic acid $\mathbf{4 a}(1.50 \mathrm{~g}, 5.6 \mathrm{mmol})$ was dissolved in $5 \mathrm{~mL} \mathrm{SOCl} \mathrm{S}_{2}$ and stirred for 6 h. The white precipitate was obtained after removed $\mathrm{SOCl}_{2}$, and then it was added to $30 \mathrm{~mL} \mathrm{CH}_{2} \mathrm{Cl}_{2}$ containing 4-dimethylaminopyridine $(0.12 \mathrm{~g}, 1.0 \mathrm{mmol})$ and triethylamine $(0.48 \mathrm{~g}, 8.0 \mathrm{mmol})$. Ethylenediamine $(0.12 \mathrm{~g}, 2.0 \mathrm{mmol})$ in 5 $\mathrm{mL} \mathrm{CH}_{2} \mathrm{Cl}_{2}$ was added by dropwise to the above solution and further stirred for $10 \mathrm{~h}$. After the solution was washed with 1 $\mathrm{mol} \cdot \mathrm{L}^{-1} \mathrm{HCl}$, saturated sodium bicarbonate and water, dried over sodium sulfate, and evaporated to remove solvent. The crude product was purified by crystallization from $\mathrm{CH}_{2} \mathrm{Cl}_{2}: n$-hexane $(V / V=1: 20)$ to give $\mathbf{L}^{R R / S S}(0.54 \mathrm{~g}, 47 \%)$. $\mathbf{L}^{R R / S S}(0.10 \mathrm{~g}$, $0.18 \mathrm{mmol})$ dissolved in $10 \mathrm{~mL}$ acetonitrile, $\mathrm{Eu}(\mathrm{OTf})_{3}(0.07 \mathrm{~g}, 0.12 \mathrm{mmol})$ in $5 \mathrm{~mL}$ acetonitrile was added dropwise to the above solution and stirred for $24 \mathrm{~h}$. After the slow volatilization of reaction solution, the white crystals were obtained, $\left[\mathrm{Eu}_{2}\left(\mathbf{L}^{R R / S S}\right)_{3}\right](\mathrm{OTf})_{6}(0.12 \mathrm{~g}, 71 \%)$. Combination of the comprehensive spectral characteristics and semiempirical geometry optimization demonstrated that the point chirality at the terminal of the ligand successfully controlled the $\Delta$ or $\Lambda$ configuration around the metal center and the $P$ or $M$ helical conformation of the helicates. The mirror-image circular dichroism (CD) and CPL spectra further confirmed the formation of the enantiomer pairs. Notably, the helicates exhibit excellent CPL emission with the $\left|g_{\text {lum }}\right|$ values reaching to 0.083 and the modest luminescence quantum yields $(\mathrm{QYs}=19 \%)$ in $\mathrm{CH}_{3} \mathrm{CN}$. This study provides an effective strategy for the syntheses of chiral lanthanide CPL materials with excellent performance.
\end{abstract}

Keywords lanthanide complex; chiral helicate; chiral induction; circularly polarized luminescence (CPL)

\section{1 引言}

手性圆偏振发光(CPL)材料由于在 3D 显示 ${ }^{[1]}$, 光数 据存储 ${ }^{[2]}$, 手性苂光探针 ${ }^{[3]}$ 以及手性传感 ${ }^{[4]}$ 等领域呈现
出广阔的应用前景而备受人们关注. 发光不对称因子 ( $g_{\text {lum }}$ 值)和发光量子产率 $(\Phi)$ 是评价 CPL 材料性能的两个 关键参数. 发光不对称因子的计算公式为 $g_{\mathrm{lum}}=2\left(I_{\mathrm{L}}-\right.$ $\left.I_{\mathrm{R}}\right) /\left(I_{\mathrm{L}}+I_{\mathrm{R}}\right)\left(\left|g_{\text {lum }}\right| \leqslant 2\right)$, 其中, $I_{\mathrm{L}}$ 和 $I_{\mathrm{R}}$ 分别为左圆偏振光

* E-mail: yanpf@vip.sina.com; lihongfeng@hlju.edu.cn

Received April 28, 2021; published June 11, 2021.

Supporting information for this article is available free of charge via the Internet at http://sioc-journal.cn.

Project supported by the National Natural Science Foundation of China (Nos. 51773054, 51872077, 52073080).

项目受国家自然科学基金(Nos. 51773054, 51872077, 52073080)资助. 
和右圆偏振光的强度. 从理论上讲, $g_{\text {lum }}=$ $4\left|m_{j i}\right| /\left|\mu_{i j}\right| \cdot \cos \theta_{\mu, m}, \mu_{i j}$ 和 $m_{j i}$ 分别为电偶极矩和磁偶极矩, $\theta_{\mu, m}$ 为二者之间的夹角. 因此, 较大的磁偶极矩 $\left|m_{j i}\right|$ 是确 保 CPL 材料具有较高 $g_{\text {lum }}$ 值的前提. 由于稀土离子的 $\mathrm{f}-\mathrm{f}$ 跃迁具有部分磁偶极跃迁性质, 这使得手性稀土 CPL 材料在理论上即能产生较强的圆偏振发射, 其 $g_{\text {lum 值通 }}$

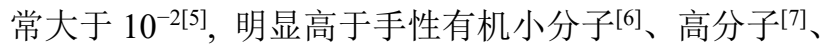
超分子 ${ }^{[8]}$ 以及过渡金属配合物 ${ }^{[9]}$ 的发光不对称度 $\left(10^{-5} \sim\right.$ $\left.10^{-3}\right)$. 例如, 2008 年, Muller 等 ${ }^{[10]}$ 合成的手性单核铕配 合物 $\mathrm{Cs}\left[\mathrm{Eu}((+)-\mathrm{hfbc})_{4}\right]$, 其 $g_{\text {lum }}$ 值高达 +1.38 , 是目前文 献报道的手性 CPL 材料在溶液态下的最高值.

发光稀土螺旋体由于兼具螺旋超分子手性和稀土 的磁偶极特性, 使之成为了一类有前途的 CPL 材料. 但 是, 稀土离子较大的半径和不稳定的配位几何为构筑光 学纯稀土螺旋体带来了较大的困难. 例如, 2015 年, Law 等 ${ }^{[11]}$ 证实如果增加配体端基手性中心与配位单元之间 的距离则会导致手性稀土螺旋体出现非对映选择性破 缺的现象. 孙庆福等 ${ }^{[12]}$ 研究发现两个相同的配位单元 之间间隔基的长度对组装体的结构具有显著的影响, 如 出现了由螺旋体向四面体或立方体的转化. 目前, 在配 体中引入手性中心是合成手性金属螺旋体最常采用的 一种方式. 但是, 通过这种方式成功构筑手性稀土螺旋 体的报道仍然十分有限。近几年, Gunnlaugsson ${ }^{[13]}$ 、 Law $^{[14]}$ 以及孙庆福 ${ }^{[15]}$ 课题组通过在双三齿配体的端基 引入手性中心的方式成功构筑了一系列手性双核稀土 螺旋体. 最近, 我们课题组 ${ }^{[16]}$ 采用在双 $\beta$-二酮配体间隔 基上引入点手性的方法, 成功预组织了配体的螺旋构 象, 使之与稀土 $\operatorname{Ln}(\mathrm{III})$ 离子在自组装时能够形成同手性 的单一构象.

通常情况下, 减小螺距能够增加螺旋体的螺旋程度
并更好地起到放大手性的作用. 鉴于此, 本工作设计了 一对以吡啶二甲酰亚胺为配位单元，亚乙基为间隔基的 手性双三齿配体 $\mathbf{L}^{R R}$ 和 $\mathbf{L}^{S S}$. 我们认为采用较短的间隔 基有利于缩短螺距，放大螺旋体的超分子手性. 实验结 果表明配体端基的点手性可以有效调控螺旋体的非对 映选择性自组装, 即一对儿对映体纯的双核铕螺旋体 $\mathrm{Eu}_{2}\left(\mathbf{L}^{R R}\right)_{3}(\mathrm{OTf})_{6}$ 和 $\mathrm{Eu}_{2}\left(\mathbf{L}^{S S}\right)_{3}(\mathrm{OTf})_{6}$ 被成功合成. 手性光 学性质研究显示螺旋体在乙腈中表现出适中的 $\left|g_{\text {lum }}\right|$ 值, 0.089 和较高的发光量子产率, $19 \%$.

\section{2 结果与讨论}

\section{1 配体及配合物的表征}

对映体纯的双三齿配体 $\mathbf{L}^{R R}$ 和 $\mathbf{L}^{S S}$ 及其配合物的合 成路线如图 1 所示. 化合物 $\mathbf{1 a} \sim \mathbf{5 a}$ 的合成参照文献报道 的方法 ${ }^{[17]}$. 配体 $\mathbf{L}^{R R}$ 和 $\mathbf{L}^{S S}$ 是通过两分子中间体 $\mathbf{5 a}$ 与一 分子乙二胺通过酰胺缩合制备得到。核磁氢谱 $\left({ }^{1} \mathrm{H}\right.$ $\mathrm{NMR})$ 、碳谱 $\left({ }^{13} \mathrm{C} \mathrm{NMR}\right)$ 以及高分辨飞行时间质谱 (ESI-TOF MS)分析证实了高纯度中间体和配体的成功 制备(图 S1～S13).

配合物是通过将配体 $\mathbf{L}^{R R / S S}$ 与稀土 $\mathrm{Ln}(\mathrm{OTf})_{3}(\mathrm{Ln}=$ $\mathrm{Eu}, \mathrm{Y}, \mathrm{Gd})$ 以 $3: 2$ 计量比在乙腈溶剂中室温搅拌获得. 螺旋体 $\left[\mathrm{Ln}_{2}\left(\mathbf{L}^{R R}\right)_{3}\right](\mathrm{OTf})_{6}$ 和 $\left[\operatorname{Ln}_{2}\left(\mathbf{L}^{S S}\right)_{3}\right](\mathrm{OTf})_{6}$ 的成功构筑 首先被 ESI-TOF MS 所证实. 如图 2a 所示, 在阳离子模 式下, 铕配合物显示出三族强度较大的离子峰, 质核比 $\mathrm{m} / \mathrm{z}$ 分别为 $573.9803,815.5809$ 和 1297.7479. 通过质核 比的计算可以将它们归属于螺旋体与抗衡阴离子三氟 甲磺酸根 $\left[\mathrm{CF}_{3} \mathrm{SO}_{3}\right]^{-}$按不同比例形成的加合物 $\left[\mathrm{Eu}_{2} \mathbf{L}_{3}+\right.$ $2 \mathrm{OTf}]^{2+} 、\left[\mathrm{Eu}_{2} \mathbf{L}_{3}+3 \mathrm{OTf}\right]^{3+}$ 和 $\left[\mathrm{Eu}_{2} \mathbf{L}_{3}+4 \mathrm{OTf}\right]^{4^{+}}$.

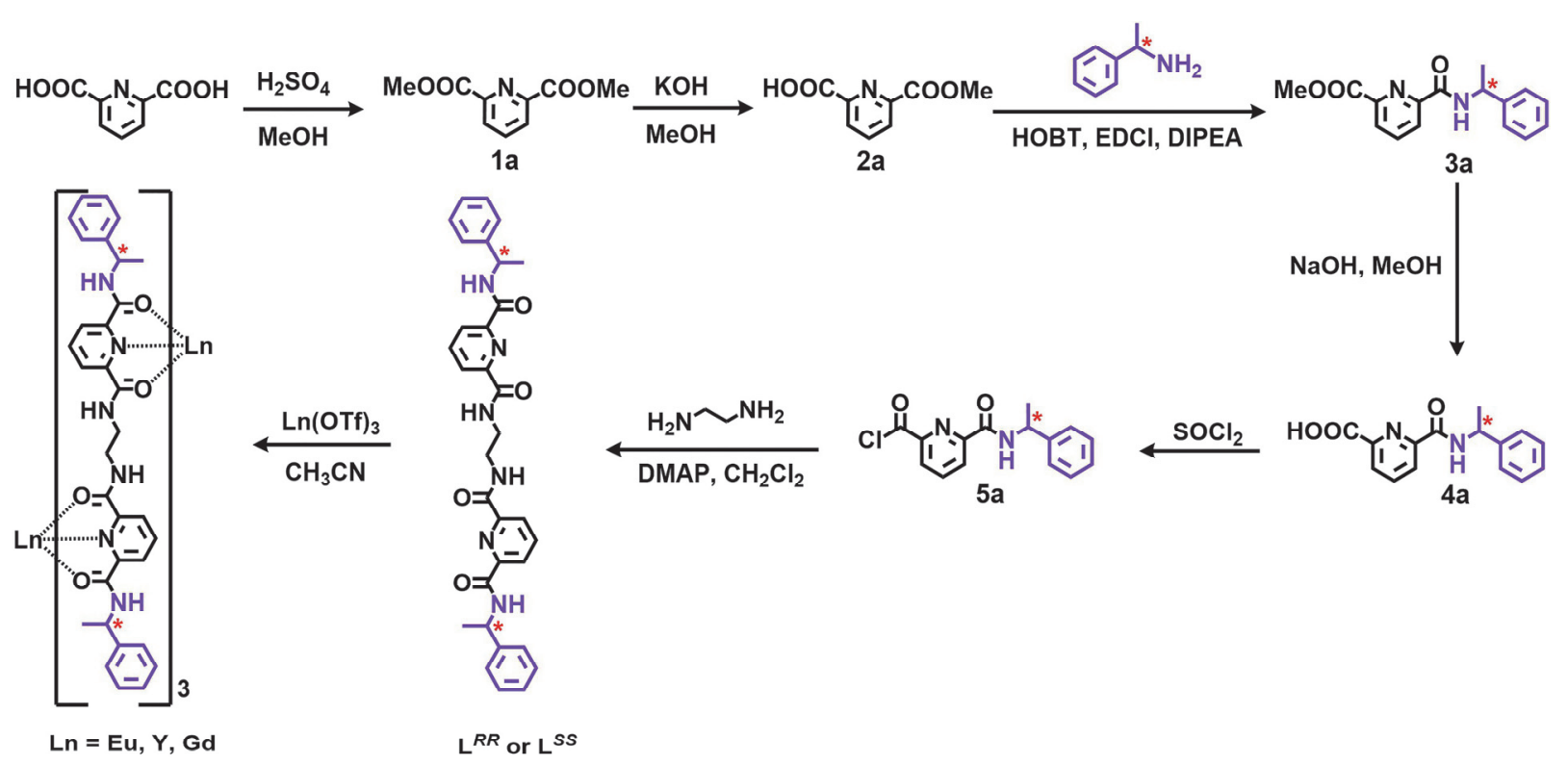

图 1 手性配体 $\mathbf{L}^{R R / S S}$ 及其稀土配合物 $\left[\mathrm{Ln}_{2}\left(\mathbf{L}^{R R / S S}\right)_{3}\right](\mathrm{OTf})_{6}$ 的合成路线

Figure 1 Syntheses of the chiral ligands $\mathbf{L}^{\text {RR/SS }}$ and the corresponding lanthanide complexes $\left[\operatorname{Ln}_{2}\left(\mathbf{L}^{R R / S S}\right)_{3}\right](\mathrm{OTf})_{6}$ 
(a)

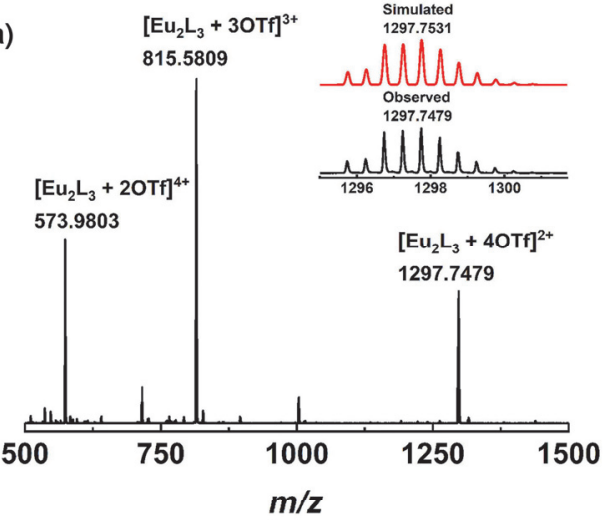

(b)
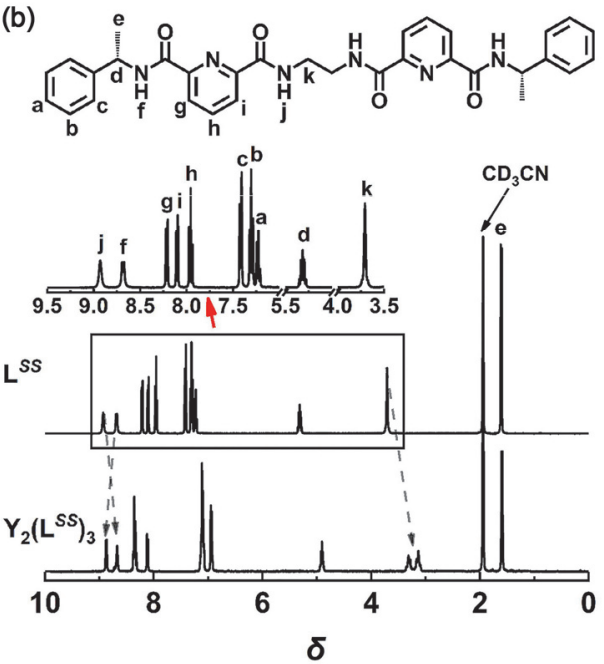

(c)

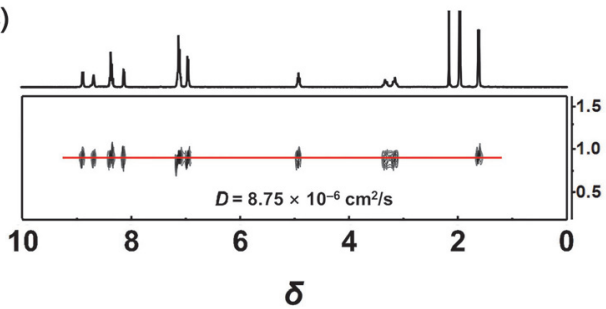

图 2 (a) $\left[\mathrm{Eu}_{2}\left(\mathbf{L}^{S S}\right)_{3}\right](\mathrm{OTf})_{6}$ 的 ESI-TOF 质谱(插图: 实测和模拟的同位 素分布). (b) $\left[\mathrm{Y}_{2}\left(\mathbf{L}^{S S}\right)_{3}\right](\mathrm{OTf})_{6}$ 在 $\mathrm{CD}_{3} \mathrm{CN}$ 中的 $400 \mathrm{MHz}{ }^{1} \mathrm{H}$ NMR. (c) $\left[\mathrm{Y}_{2}\left(\mathbf{L}^{S S}\right)_{3}\right](\mathrm{OTf})_{6}$ 在 $\mathrm{CD}_{3} \mathrm{CN}$ 中的 ${ }^{1} \mathrm{H}$ DOSY NMR

Figure 2 (a) ESI-TOF mass spectrum of $\left[\mathrm{Eu}_{2}\left(\mathbf{L}^{S S}\right)_{3}\right](\mathrm{OTf})_{6}$ with insets showing the simulated (Sim.) and observed (Obs.) isotopic pattern. (b) ${ }^{1} \mathrm{H}$ NMR $(400 \mathrm{MHz})$ spectra of free ligand $\mathbf{L}^{S S}$ and $\left[\mathrm{Y}_{2}\left(\mathbf{L}^{S S}\right)_{3}\right](\mathrm{OTf})_{6}$ in $\mathrm{CD}_{3} \mathrm{CN}$. (c) ${ }^{1} \mathrm{H}$ DOSY NMR spectrum of $\left[\mathrm{Y}_{2}\left(\mathbf{L}^{S S}\right)_{3}\right](\mathrm{OTf})_{6}$ in $\mathrm{CD}_{3} \mathrm{CN}$

为了进一步验证归属的准确性, 我们对 $m / z=$ 1297.7479 的离子峰进行了同位素分布分析, 结果显示 理论值与实测值能够完美的吻合, 这表明离子峰归属的 准确性, 并证实了螺旋体的形成. 同样, 钎 $(\mathrm{Y})$ 和钝 $(\mathrm{Gd})$ 配合物的质谱也证实了三股螺旋体的形成(图 S14 S18).

此外, 我们也对配合物进行了核磁共振波谱表征. 为了避免由于 $\mathrm{Eu}^{3+}$ 离子的顺磁性所引起的共振峰展宽 而导致的分辨率降低, 我们利用稀土钎配合物 $\mathrm{Y}_{2}\left(\mathbf{L}^{R R / S S}\right)_{3}$ 替代 $\mathrm{Eu}_{2}\left(\mathbf{L}^{R R / S S}\right)_{3}$ 进行了核磁氢谱( $\left.{ }^{1} \mathrm{H} \mathrm{NMR}\right)$ 的
研究(图 S19 S20). 从图 2b 中我们能够清楚地观测到 一组清晰尖锐的信号峰, 并且与自由配体 $\mathbf{L}$ 相比没有表 现出明显的峰展宽迹象. 不同的是大部分信号峰向高场 发生了位移. 半峰宽较窄且没有出现新的归属于非对映 异构体的信号, 说明在溶液体系下配合物具有较高的非 对映体纯度. 这也意味着同手性螺旋体的成功构筑和配 体端基点手性在调控组装体非对映选择性上的有效性. 此外，配合物在乙腈中的扩散排序核磁共振谱(DOSY) 也只显示一组扩散系数为 $D=8.75 \times 10^{-6} \mathrm{~cm}^{2} \cdot \mathrm{s}^{-1}$ 的信号 (图 2c), 该结果说明手性配体与稀土离子在自组装过程 中克服了熵驱动产生的由多种非对映异构体组成混合 物的问题, 进一步验证了配合物具有高的非对映体纯 度, 只形成了单一物种.

\section{2 配合物 $\left[\mathrm{Eu}_{2}\left(\mathrm{~L}^{R R}\right)_{3}\right]^{6+}$ 和 $\left[\mathrm{Eu}_{2}\left(\mathrm{~L}^{\mathrm{SS}}\right)_{3}\right]^{6+}$ 的构型优化}

为了更好地理解配合物的螺旋结构, 我们通过 LUMPAC 3.0 软件中的 Sparkle/RM1 模式对配合物的结 构进行了半经验量化计算 ${ }^{[18]}$. 计算结果显示配体端基 的点手性可以有效地控制组装体在自组装过程中的立 体选择性(图 3). 在螺旋体 $\left[\mathrm{Eu}_{2}\left(\mathbf{L}^{R R}\right)_{3}\right]^{6+}$ 中, 配体端基 $R$ 构型的手性碳调控了三个三齿配位单元围绕稀土 $\mathrm{Eu}^{3+}$ 离子形成左手螺旋 $(M)$ 构象, 并赋予金属中心 $\Lambda \Lambda$ 构型; 相反, $R$ 构型的手性碳则调控了右手 $P$ 螺旋体的形成和 金属中心的 $\Delta \Delta$ 构型. 结构分析显示螺旋体具有 $C_{3}$ 对称 性, 且螺旋体的每一个 $\mathrm{Eu}^{3+}$ 离子与三股配体上的六个 $\mathrm{O}$ 原子和三个 $\mathrm{N}$ 原子形成饱和的九配位环境. 利用 SHAPE 2.1 软件, 我们对 $\mathrm{Eu}^{3+}$ 离子周围的配位几何构型 进行了计算, 结果显示金属中心的配位构型为三帽三棱 柱(图 S21).
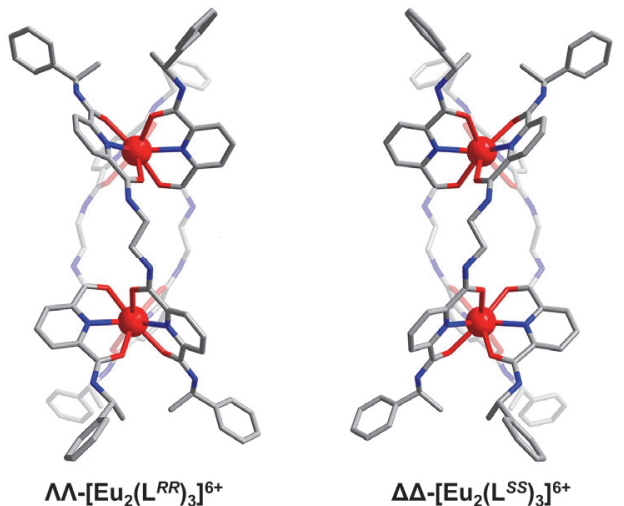

图 3 Sparkle/AM1 优化的配 合物 $\left[\mathrm{Eu}_{2}\left(\mathbf{L}^{R R}\right)_{3}\right](\mathrm{OTf})_{6}($ 左 ) 和 $\left[\mathrm{Eu}_{2}\left(\mathbf{L}^{S S}\right)_{3}\right](\mathrm{OTf})_{6}$ (右)的基态构型

Figure 3 Sparkle/AM1 ground state geometries of the complexes $\left[\mathrm{Eu}_{2}\left(\mathbf{L}^{R R}\right)_{3}\right](\mathrm{OTf})_{6}$ (left) and $\left[\mathrm{Eu}_{2}\left(\mathbf{L}^{S S}\right)_{3}\right](\mathrm{OTf})_{6}$ (right)

\section{3 配合物在溶液中的种类和热力学稳定性}

考虑到配合物在溶液中存在解离平衡, 我们通过紫 外-可见光谱滴定实验对配合物在平衡状态下可能存在 的组装体种类和热力学稳定性进行了研究. 首先, 我们 配制了配体浓度为 $3 \times 10^{-5} \mathrm{~mol} \cdot \mathrm{L}^{-1}$ 的乙腈溶液, 然后监 
测了该溶液在滴加不同计量比 $\mathrm{Eu}(\mathrm{OTf})_{3}$ 乙腈溶液 $(3 \times$ $\left.10^{-5} \mathrm{~mol} \cdot \mathrm{L}^{-1},[\mathrm{Eu}] /\left[\mathbf{L}^{S S}\right]=0 \sim 2\right)$ 的紫外吸收光谱变化. 如 图 4 所示, 随着 $\mathrm{Eu}^{3+}$ 离子浓度的增加, 配体 $\mathbf{L}^{S S}$ 在 229 $\mathrm{nm}$ 处的吸收逐渐增强, 而位于 267、274 和 $284 \mathrm{~nm}$ 的吸 收不但出现了增强, 而且出现了约 $2 \sim 4 \mathrm{~nm}$ 的轻微红移 (图 4a).
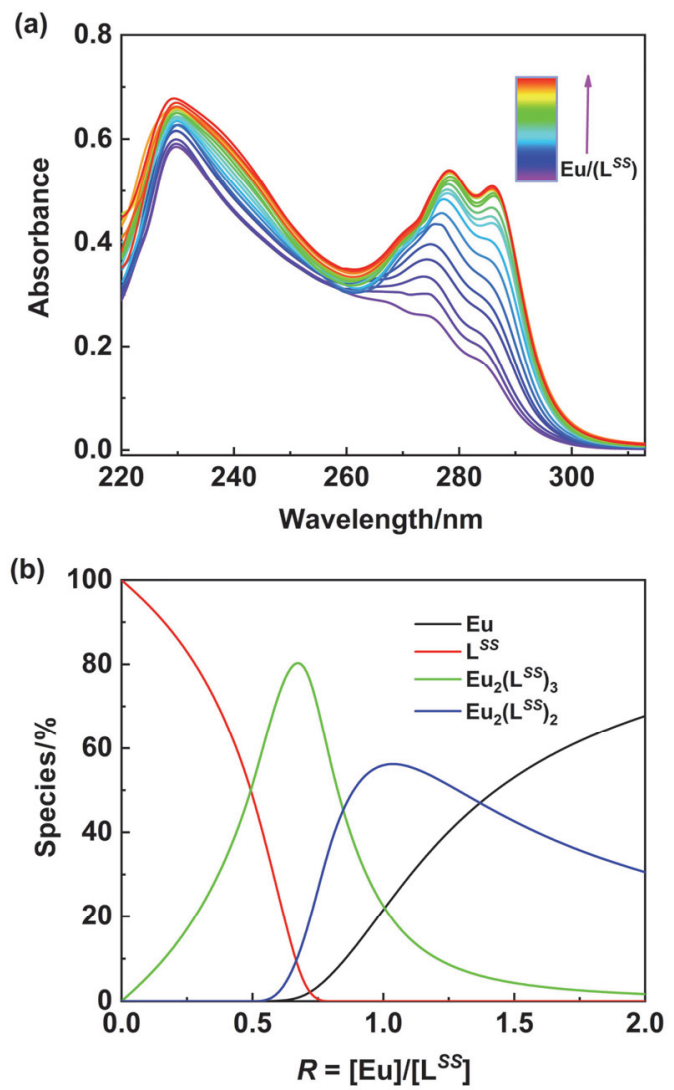

图 4 (a) 配体 $\mathbf{L}^{S S}$ 在 $\mathrm{CH}_{3} \mathrm{CN}\left(3 \times 10^{-5} \mathrm{~mol} \cdot \mathrm{L}^{-1}\right)$ 中的 $\mathrm{UV}$-vis 光谱随着 $0 \sim 2.0$ equiv. $\mathrm{Eu}\left(\mathrm{CF}_{3} \mathrm{SO}_{3}\right)_{3}\left(\mathrm{CH}_{3} \mathrm{CN}, 3 \times 10^{-3} \mathrm{~mol} \cdot \mathrm{L}^{-1}\right)$ 加入的变化. (b) 计算的物种分布图

Figure 4 (a) Variation of the UV-vis spectra of $\mathbf{L}^{S S}$ in $\mathrm{CH}_{3} \mathrm{CN}\left(3 \times 10^{-5}\right.$ $\left.\mathrm{mol} \cdot \mathrm{L}^{-1}\right)$ upon addition of the $\mathrm{Eu}\left(\mathrm{CF}_{3} \mathrm{SO}_{3}\right)_{3}\left(\mathrm{CH}_{3} \mathrm{CN}, 3 \times 10^{-3} \mathrm{~mol} \cdot \mathrm{L}^{-1}\right)$ from $0 \sim 2.0$ equiv. (b) Calculated distribution plots of the species

随后，我们利用 “Jplus ReactLab Equilibria” 软件对 上述光谱数据进行了最小二乘法拟合. 数据分析结果显 示在滴定过程中物种 $\mathrm{Eu}_{2}\left(\mathbf{L}^{S S}\right)_{3}$ 和 $\mathrm{Eu}_{2}\left(\mathbf{L}^{S S}\right)_{2}$ 在溶液中的 存在是合理的，其配位解离平衡方程可以表示为:

$$
\begin{aligned}
& 2 \mathrm{Eu}+3 \mathrm{~L} \rightleftharpoons \mathrm{Eu}_{2} \mathrm{~L}_{3} \quad \log \beta_{23} \\
& 2 \mathrm{Eu}+2 \mathrm{~L} \rightleftharpoons \mathrm{Eu}_{2} \mathrm{~L}_{2} \quad \log \beta_{22}
\end{aligned}
$$

根据拟合数据我们得到了物种分布随计量比 $R=$ $[\mathrm{Eu}] /\left[\mathbf{L}^{S S}\right]$ 的变化曲线, 如图 $4 \mathrm{~b}$. 在计量比 $R=0.66$ 时, 体系中出现最优物种 $\mathrm{Eu}_{2}\left(\mathbf{L}^{S S}\right)_{3}$, 含量高达 $81 \%$. 两个物 种的累积稳定常数以及在 $R=0.66$ 时的物种浓度和百分 含量总结在表 1 中. 此外, 我们还监测了 $\mathrm{Y}(\mathrm{OTf})_{3}$ 对配 体 $\mathbf{L}^{S S}$ 乙腈溶液 $\left(1.77 \times 10^{-2} \mathrm{~mol} \cdot \mathrm{L}^{-1},[\mathrm{Y}] /\left[\mathbf{L}^{S S}\right]=0 \sim 2\right)$ 的 核磁滴定过程, 随着稀土离子的逐渐加入, 配体的核磁
信号峰发生了明显的位移，直到 $[\mathrm{Y}] /\left[\mathbf{L}^{S S}\right]=0.66$ 时，核 磁光谱信号峰不再发生明显变化(图 S22), 且当体系中 存在大量稀土离子时, 未见明显新物种的生成, 以上结 果均表明形成了热力学稳定的稀土螺旋体.

表 1 通过拟合吸收光谱变化计算的物种累积平衡常数、种类分布以 及在 $[\mathrm{Eu}] /\left[\mathrm{L}^{S S}\right]=0.66$ 时计算的物种浓度

Table 1 Binding constants, species distribution estimated by fitting the changes in absorption spectra, and the calculated species concentration of the complexes with the $[\mathrm{Eu}] /\left[\mathbf{L}^{S S}\right]=0.66$

\begin{tabular}{cccc}
\hline Species & $\log \beta_{\mathrm{M} / \mathrm{L}}$ & $\begin{array}{c}\text { \% Species for } \\
\mathrm{M} /\left[\mathbf{L}^{S S}\right]\end{array}$ & $\begin{array}{c}\text { Concentration of } \\
\text { Species } /\left(10^{-6}\right. \\
\left.\mathrm{mol} \cdot \mathrm{L}^{-1}\right)\end{array}$ \\
\hline $\log \beta_{23}$ & 36.600 & 81.0 & 0.92 \\
$\log \beta_{22}$ & 26.636 & 9.5 & 0.11 \\
\hline
\end{tabular}

\section{4 配合物的手性光学性质}

基于上述分析，我们可以证实点手性可以有效调控 对映体纯稀土螺旋体的生成. 为了研究配体端基的点手 性对配合物超分子手性的影响, 我们首先对手性配体 $\mathbf{L}^{R R}$ 和 $\mathbf{L}^{S S}$ 及其配合物 $\left[\mathrm{Eu}_{2}\left(\mathbf{L}^{R R}\right)_{3}\right](\mathrm{OTf})_{6}$ 和 $\left[\mathrm{Eu}_{2}\left(\mathbf{L}^{S S}\right)_{3}\right]-$ $(\mathrm{OTf})_{6}$ 进行了圆二色(CD)光谱的测试. 如图 5a 所示, 配 体及其配合物在乙腈中展现出两对完美的镜像 CD 信 号. 手性配体 $\mathbf{L}^{R R}$ 和 $\mathbf{L}^{S S}$ 在 $250 \sim 350 \mathrm{~nm}$ 范围内分别显
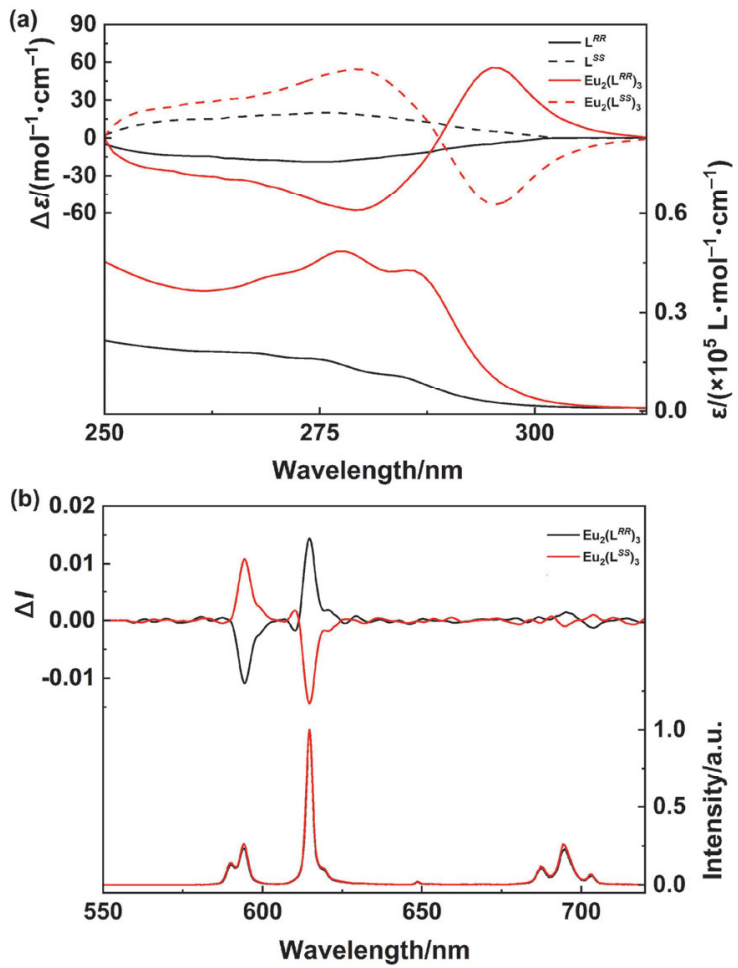

图 5 (a) 手性配体 $\mathbf{L}^{R R / S S}$ 及稀土螺旋体 $\left[\mathrm{Eu}_{2}\left(\mathbf{L}^{R R}\right)_{3}\right](\mathrm{OTf})_{6}$ 和 $\left[\mathrm{Eu}_{2}\left(\mathbf{L}^{S S}\right)_{3}\right](\mathrm{OTf})_{6}$ 在乙腈中的 UV-vis 光谱 $(下)$ 和 $\mathrm{CD}$ 光谱 $\left(\right.$ 上) $\left(1.0 \times 10^{-5}\right.$ $\left.\mathrm{mol} \cdot \mathrm{L}^{-1}\right)$. (b) 手性稀土螺旋体 $\left[\mathrm{Eu}_{2}\left(\mathbf{L}^{R R}\right)_{3}\right](\mathrm{OTf})_{6}$ 和 $\left[\mathrm{Eu}_{2}\left(\mathbf{L}^{S S}\right)_{3}\right](\mathrm{OTf})_{6}$ 在 乙腈中的发射光谱(下)和 $\mathrm{CPL}$ 光谱(上) $\left(1.0 \times 10^{-5} \mathrm{~mol} \cdot \mathrm{L}^{-1}\right)$

Figure 5 (a) UV-vis absorption (lower curves) and CD spectra (upper curves) of the chiral ligands $\mathbf{L}^{R R / S S}$ and lanthanide helicates $\left[\mathrm{Eu}_{2}\left(\mathbf{L}^{R R}\right)_{3}\right](\mathrm{OTf})_{6}$ and $\left[\mathrm{Eu}_{2}\left(\mathbf{L}^{S S}\right)_{3}\right](\mathrm{OTf})_{6}$ in $\mathrm{CH}_{3} \mathrm{CN}\left(1.0 \times 10^{-5} \mathrm{~mol} \cdot \mathrm{L}^{-1}\right)$. (b) Total luminescence (lower curves) and CPL spectra (upper curves) of $\left[\mathrm{Eu}_{2}\left(\mathbf{L}^{R R}\right)_{3}\right](\mathrm{OTf})_{6}$ and $\left[\mathrm{Eu}_{2}\left(\mathbf{L}^{S S}\right)_{3}\right](\mathrm{OTf})_{6}$ in $\mathrm{CH}_{3} \mathrm{CN}\left(1.0 \times 10^{-5} \mathrm{~mol} \cdot \mathrm{L}^{-1}\right)$ 
示出一个正的和一个负的 Cotton 效应. 与手性配体不同 的是螺旋体 $\left[\mathrm{Eu}_{2}\left(\mathbf{L}^{R R}\right)_{3}\right](\mathrm{OTf})_{6}$ 和 $\left[\mathrm{Eu}_{2}\left(\mathbf{L}^{S S}\right)_{3}\right](\mathrm{OTf})_{6}$ 显示出 交叉点位于 $288 \mathrm{~nm}$ 的激子耦合效应, 其达维多夫分裂 达到 $16 \mathrm{~nm}$. 激子偶合的出现可归因于螺旋体中三股配 体的生色团在空间上距离的拉近 ${ }^{[19]}$. 此外, 在 UV 光谱 中, 我们观察到螺旋体的最大紫外吸收波长相比于配体 红移了 $3 \mathrm{~nm}$. 这很可能来源于稀土 $\mathrm{Eu}^{3+}$ 离子的吸电子 效应, 其促使配体内发生轻微的电荷转移, 并因而降低 了基态电子跃迁所需的能量.

通过观察 $\mathrm{CD}$ 光谱激子偶合的样式和金属中心的构 型, 人们总结出了一个经验规律 ${ }^{[20]}$ : 即, 正激子耦合对 应于金属中心的 $\Lambda$ 构型; 相反, 负激子耦合对应于金属 中心的 $\Delta$ 构型. 根据该规则, 螺旋体 $\left[\mathrm{Eu}_{2}\left(\mathbf{L}^{R R}\right)_{3}\right](\mathrm{OTf})_{6}$ 和 $\left[\mathrm{Eu}_{2}\left(\mathbf{L}^{S S}\right)_{3}\right](\mathrm{OTf})_{6}$ 的中心稀土 $\mathrm{Eu}^{3+}{ }^{+}$离子应分别采取 $\Lambda \Lambda$ 和 $\Delta \Delta$ 构型. 这一推断与半经验量化计算得到的金属构 型相一致. 值得注意的是, 螺旋体还表现出明显高于配 体的摩尔 $\mathrm{ECD}$ 值 $(\Delta \varepsilon)$, 这说明螺旋结构的形成有效地实 现了对配体手性的放大. 该结果再一次证实了配体端基 的点手性具有控制形成同手性稀土螺旋体的能力, 并使 其光学活性显著增强.

图 $5 \mathrm{~b}$ 显示了配 合物 $\left[\mathrm{Eu}_{2}\left(\mathbf{L}^{R R}\right)_{3}\right](\mathrm{OTf})_{6}$ 和 $\left[\mathrm{Eu}_{2}\left(\mathbf{L}^{S S}\right)_{3}\right](\mathrm{OTf})_{6}$ 在乙腈中发射光谱和 $\mathrm{CPL}$ 光谱. 在发 射光谱中, 我们能够清楚地观察到来自于中心稀土 $\mathrm{Eu}^{3+}$ 离子的特征发射, 对应于其 ${ }^{5} \mathrm{D}_{0} \rightarrow{ }^{7} \mathrm{~F}_{J}(J=0 \sim 4)$ 跃 迁. 通过监测 $\mathrm{Eu}^{3^{+}}$离子在 $612 \mathrm{~nm}$ 处 ${ }^{5} \mathrm{D}_{0} \rightarrow{ }^{7} \mathrm{~F}_{2}$ 的跃迁, 我 们得到了配合物的激发光谱(图 S23). 从图中我们能够 看到配合物的最大激发波长为 $278 \mathrm{~nm}$. 随后, 我们以 $\mathrm{Cs}_{3}\left[\mathrm{Eu}(\mathrm{dpa})_{3}\right] \cdot 9 \mathrm{H}_{2} \mathrm{O}$ 配合物作为标准样品, 通过外标法 计算得到了配合物 $\left[\mathrm{Eu}_{2}\left(\mathrm{~L}^{R R}\right)_{3}\right](\mathrm{OTf})_{6}$ 和 $\left[\mathrm{Eu}_{2}\left(\mathrm{~L}^{S S}\right)_{3}\right](\mathrm{OTf})_{6}$ 的发光量子产率为 $19 \%$.

在 CPL 光谱中, 螺旋体同样呈现出了一对镜像的 CPL 信号, 这进一步说明了对映体的形成. 谱图显示螺 旋体在 594 和 $612 \mathrm{~nm}$ 处具有较强的 CPL 信号, 其分别 归属于 ${ }^{5} \mathrm{D}_{0} \rightarrow{ }^{7} \mathrm{~F}_{1}$ 的磁偶极跃迁和 ${ }^{5} \mathrm{D}_{0} \rightarrow{ }^{7} \mathrm{~F}_{2}$ 的电偶极跃 迁. 从理论上来说, 磁偶极跃迁允许产生较大的不对称 因子 $\left(g_{\text {lum }}\right)$. 测试结果显示螺旋体 $\left[\mathrm{Eu}_{2}\left(\mathbf{L}^{R R}\right)_{3}\right](\mathrm{OTf})_{6}$ 和 $\left[\mathrm{Eu}_{2}\left(\mathbf{L}^{S S}\right)_{3}\right](\mathrm{OTf})_{6}$ 在 $594 \mathrm{~nm}$ 处的 $\left|g_{\text {lum }}\right|$ 值可达 0.083 . 该值 与已报道的基于吡啶二羧酸类稀土配合物的 $\left|g_{\mathrm{lum}}\right|$ 值类 似 ${ }^{[21]}$. 螺旋体中 $\mathrm{Eu}^{3+}$ 离子 ${ }^{5} \mathrm{D}_{0} \rightarrow{ }^{7} \mathrm{~F}_{J}(J=0 \sim 4)$ 跃迁的具 体 $g_{\text {lum }}$ 值总结在表 2 中. 此外, 手性稀土配合物的 CPL 光谱信号样式与稀土 $\mathrm{Ln}^{3}$ 离子的绝对构型也具有一定 相关性. 根据 Muller 和我们以前的报道所总结的经验规 律 ${ }^{[5,22]}$, 在 ${ }^{5} \mathrm{D}_{0} \rightarrow{ }^{7} \mathrm{~F}_{2}$ 跃迁和 ${ }^{5} \mathrm{D}_{0} \rightarrow{ }^{7} \mathrm{~F}_{1}$ 跃迁处分别显示为 正和负信号, 代表手性稀土配合物的 $\mathrm{Eu}^{3+}$ 离子具有 $\Lambda$ 构 型; 与之相反, 则具有 $\Delta$ 构型. 依据该规律我们也能推 断 $\mathrm{Eu}^{3+}$ 离子在 $\left[\mathrm{Eu}_{2}\left(\mathbf{L}^{R R}\right)_{3}\right](\mathrm{OTf})_{6}$ 中为 $\Lambda \Lambda$ 构型, 而在 $\left[\mathrm{Eu}_{2}\left(\mathbf{L}^{S S}\right)_{3}\right](\mathrm{OTf})_{6}$ 中为 $\Delta \Delta$ 构型. 这一推断与我们在 $\mathrm{CD}$ 光谱和量化计算中所得的结果一致.

\section{5 配合物的光物理性质}

发光量子产率 $(\mathrm{QY})$ 是评价手性 CPL 材料发光性能 的另一个重要参数. 外标法计算得到配合物的发光量子 产率可达 19\%. 根据公式(3), 稀土配合物发光量子产率 的大小取决于稀土 $\mathrm{Ln}^{3+}$ 离子发射的内量子效率 $\left(\Phi_{\mathrm{Ln}}\right)$ 和 配体对稀土离子的能量传递效率 $\left(\eta_{\mathrm{sen}}\right)$. 为了阐明这两个 因素对螺旋体发光量子产率的影响, 我们首先根据公式 (4)和公式(5)计算了稀土 $\mathrm{Eu}^{3+}$ 离子发射的内量子效率 $\left(\Phi_{\mathrm{Ln}}\right)$. 其中, $\tau_{\mathrm{rad}}$ 为辐射寿命, $\tau_{\mathrm{obs}}$ 为测试的 $\mathrm{Eu}^{3+}$ 的寿命, $A_{\mathrm{MD}, 0}$ 为磁偶极 ${ }^{5} \mathrm{D}_{0} \rightarrow{ }^{7} \mathrm{~F}_{1}$ 自发跃迁辐射概率, $n$ 为溶剂的 折射率, $I_{\mathrm{tot}}$ 为 ${ }^{5} \mathrm{D}_{0} \rightarrow{ }^{7} \mathrm{~F}_{\mathrm{J}}$ 跃迁的总积分面积, $I_{\mathrm{MD}}$ 为 ${ }^{5} \mathrm{D}_{0} \rightarrow{ }^{7} \mathrm{~F}_{1}$ 跃迁的积分面积. 螺旋体在乙腈中的光物理数 据总结在表 2 中.

$$
\begin{aligned}
& \Phi_{\text {overall }}=\eta_{\mathrm{sen}} \Phi_{\mathrm{Ln}} \\
& \Phi_{\mathrm{Ln}}=\frac{k_{\mathrm{r}}}{k_{\mathrm{r}}+k_{\mathrm{nr}}}=\frac{\tau_{\text {obs }}}{\tau_{\mathrm{rad}}} \\
& k_{\mathrm{r}}=\frac{1}{\tau_{\mathrm{rad}}}=A_{\mathrm{MD}, 0} n^{3}\left(\frac{I_{\mathrm{tot}}}{I_{\mathrm{MD}}}\right)
\end{aligned}
$$

根据计算得到的辐射寿命 $\tau_{\mathrm{rad}}$ 和观察到的寿命 $\tau_{\mathrm{obs}}$ (图 S24 S25), 我们计算得到了稀土 $\mathrm{Eu}^{3+}$ 离子发射的内 量子效率 $\left(\Phi_{\mathrm{Eu}}\right)$ 为 $25 \%$. 在此基础上, 根据公式(4), 稀土 $\mathrm{Eu}^{3+}$ 离子的内量子效率取决于配合物辐射跃迁与非辐 射跃迁过程的竞争. 计算结果显示配合物的辐射速率常 数 $\left(k_{\mathrm{r}}\right)$ 和非辐射速率常数 $\left(k_{\mathrm{nr}}\right)$ 分别为 $180 \mathrm{~s}^{-1}$ 和 $551 \mathrm{~s}^{-1}$. 一 般情况下, 辐射跃迁速率常数的大小与稀土 $\mathrm{Ln}^{3+}$ 离子 的配位几何构型有关. 根据我们及其他课题组已报道的 研究结果 ${ }^{[23]}$, 辐射速率常数通常会随着稀土 $\mathrm{Ln}^{3+}$ 离子 周围配位几何构型对称性的升高而降低. 因此, 我们认 为较低的辐射速率常数可能来源于 $\mathrm{Eu}^{3+}$ 离子周围较高 的对称性，这与我们计算得到的三帽三棱柱(9-TTP)的 配位构型一致. 最后，根据公式(3)计算得到了配合物的 能量传递效率 $\left(\eta_{\mathrm{sen}}\right)$ 高达 $75 \%$. 根据 Latva 经验规则, 配 体的三线态能级 $\left(\mathrm{T}_{1}\right)$ 与稀土 $\mathrm{Ln}^{3+}$ 离子的激发态能级 $\left({ }^{5} \mathrm{D}_{0}\right)$ 差决定了配合物的能量传递效率. 通常情况下, 能级差 $\Delta E\left(\mathrm{~T}_{1}{ }^{-5} \mathrm{D}_{0}\right)$ 在 $2500 \sim 5000 \mathrm{~cm}^{-1}$ 是确保配体到稀土 $\mathrm{Ln}^{3+}$ 离子高效能量传递的最佳范围 [24]. 我们通过配合物磷 光光谱的最大发射 $(\lambda=437 \mathrm{~nm})$ 计算得到了配体的三线 态能级为 $22883 \mathrm{~cm}^{-1}$ (图 $\mathrm{S} 26$ ), 其与稀土离子 ${ }^{5} \mathrm{D}_{0}$ 能级 $\left(17500 \mathrm{~cm}^{-1}\right)$ 之间的能级差 $\Delta E$ 为 $5383 \mathrm{~cm}^{-1}$, 该值非常 接近于最佳的能级差范围. 因此, 我们认为螺旋体良好 的发光量子产率主要取决于配体对稀土 $\mathrm{Eu}^{3+}$ 离子的高 效能量传递. 
表 2 辐射 $\left(k_{\mathrm{r}}\right)$ 和非辐射 $\left(k_{\mathrm{n}}\right)$ 跃迁的速率常数, 观察的发光寿命 $\left(\tau_{\mathrm{Eu} \mathrm{obs}}\right)$, 敏化效率 $\left(\eta_{\mathrm{sens}}\right)$, 固有量子产率 $\left(\Phi_{\mathrm{Eu}}\right)$, 总发光量子产率 $\left(\Phi_{\mathrm{overall}}\right)$ 和 $\mathrm{Eu} \mathrm{u}^{3+}$ 离子的 ${ }^{5} \mathrm{D}_{0} \rightarrow{ }^{7} \mathrm{~F}_{J}$ 的 $g_{\text {lum }}$ 值 ${ }^{a}$

Table 2 Rate constants of radiative $\left(k_{\mathrm{r}}\right)$ and nonradiative $\left(k_{\mathrm{nr}}\right)$ decay, observed luminescence lifetime $\left(\tau_{\mathrm{Eu} \mathrm{obs}}\right)$, sensitization efficiency $\left(\eta_{\text {sens }}\right)$, intrinsic quantum yields $\left(\Phi_{\mathrm{Eu}}\right)$, overall quantum yield $\left(\Phi_{\text {overall }}\right)$, and $g_{\text {lum }}$ values for ${ }^{5} \mathrm{D}_{0} \rightarrow{ }^{7} \mathrm{~F}_{J}$ of the $\mathrm{Eu}^{3+}$ ion ${ }^{a}$

\begin{tabular}{|c|c|c|c|c|c|c|c|c|c|c|c|}
\hline \multirow{2}{*}{ Complex } & \multirow{2}{*}{$k_{\mathrm{r}} / \mathrm{s}^{-1}$} & \multirow{2}{*}{$k_{\mathrm{nr}} / \mathrm{s}^{-1}$} & \multirow{2}{*}{$\tau_{\mathrm{obs}} / \mu \mathrm{s}$} & \multirow{2}{*}{$\Phi_{\mathrm{Eu}} / \%$} & \multirow{2}{*}{$\eta_{\text {sens }} / \%$} & \multirow{2}{*}{$\Phi_{\text {overall }} / \%$} & \multicolumn{5}{|c|}{$g_{\text {lum }}{ }^{5} \mathrm{D}_{0} \rightarrow{ }^{7} \mathrm{~F}_{J}(J=0,1,2,3,4)$} \\
\hline & & & & & & & $J=0$ & $J=1$ & $J=2$ & $J=3$ & $J=4$ \\
\hline $\mathrm{Eu}_{2}\left(\mathbf{L}^{R R}\right)_{3}$ & 180 & 551 & 1372 & 24.8 & 75.0 & 18.6 & -0.002 & -0.083 & 0.026 & 0.037 & 0.011 \\
\hline $\mathrm{Eu}_{2}\left(\mathbf{L}^{S S}\right)_{3}$ & 184 & 553 & 1356 & 24.9 & 75.9 & 18.9 & 0.002 & 0.083 & -0.024 & -0.034 & -0.013 \\
\hline
\end{tabular}

${ }^{a} \tau_{\mathrm{obs}}$ 误差为 $\pm 0.05 \mathrm{~ms}$, 其他参数误差在 $10 \%$ 以内, $\lambda_{\mathrm{ex}}=278 \mathrm{~nm}$.

\section{3 结论}

成功合成了一对具有良好圆偏振发光(CPL)性质的 对映体纯双核稀土螺旋体. 全面的光谱分析和半经验量 化计算证实了配体端基的点手性能够有效控制同手性 稀土螺旋体的形成. 具有 $R$ 构型点手性的配体能够与稀 土离子形成具有 $M$ 螺旋构象的同手性双核稀土螺旋体 $\Lambda \Lambda-\left[\mathrm{Eu}_{2}\left(\mathbf{L}^{R R}\right)_{3}\right](\mathrm{OTf})_{6}$, 而 $S$ 构型点手性能够调控 $\Delta \Delta-\left[\mathrm{Eu}_{2}\left(\mathbf{L}^{S S}\right)_{3}\right](\mathrm{OTf})_{6}$ 的形成. $\mathrm{CD}$ 光谱显示螺旋体的形成 放大了配体的基态手性，并为激发态手性的凸显提供了 保障. CPL 测试显示螺旋体能够发射出适中的圆偏振光, 发光不对称因子 $\left|g_{\text {lum }}\right|$ 能够达到 0.083 . 此外, 配合物也表 现出了 $19 \%$ 的发光量子产率. 因此, 该研究为构筑同手 性稀土超分子 CPL 材料提供了一个可行性参考.

\section{4 实验部分}

\section{$4.11 \mathrm{a}$ 的合成}

将 2,6-吡啶二甲酸 $(5.00 \mathrm{~g}, 30.0 \mathrm{mmol}$ )溶解在装有 $80 \mathrm{~mL} \mathrm{CH} \mathrm{CH}_{3} \mathrm{OH}$ 的圆底烧瓶中, 向该溶液中缓慢滴加 2.5 $\mathrm{mL}$ 浓 $\mathrm{H}_{2} \mathrm{SO}_{4}$. 反应体系加热回流 $24 \mathrm{~h}$, 并用薄层色谱点 样分析法(TLC)监测反应过程. 反应结束后, 减压蒸馏 除去溶剂, 得到白色固体 $5.75 \mathrm{~g}$, 产率为 $98 \%$.

\section{$4.22 a$ 的合成}

在 $0{ }^{\circ} \mathrm{C}$ 条件下, 将 $1 \mathrm{a}(1.16 \mathrm{~g}, 6.0 \mathrm{mmol})$ 溶解在装有 $25 \mathrm{~mL} \mathrm{CH} \mathrm{CH}_{3} \mathrm{OH}$ 的圆底烧瓶中, 向该反应体系中加入 $\mathrm{KOH}(0.33 \mathrm{~g}, 6.0 \mathrm{mmol})$ 并持续搅拌 $12 \mathrm{~h}$, 通过 TLC 监测 反应过程. 反应结束后, 减压蒸馏除去 $\mathrm{CH}_{3} \mathrm{OH}$, 得到白 色固体. 随后, 用乙酸乙酯淋洗白色固体除去残余的原 料. 将白色固体重新溶解在 $25 \mathrm{~mL}$ 水中, 用 $1 \mathrm{~mol} \cdot \mathrm{L}^{-1}$ $\mathrm{HCl}$ 调节水溶液 $\mathrm{pH}=2$, 乙酸乙酯 $(25 \mathrm{~mL} \times 3)$ 萃取, 合 并有机层并用无水 $\mathrm{Na}_{2} \mathrm{SO}_{4}$ 干燥. 减压蒸馏除去乙酸乙 酯, 得到白色固体 $0.80 \mathrm{~g}$, 产率为 $73 \%$.

\section{$4.33 a$ 和 $4 a$ 的合成}

将 $2 \mathrm{a}(1.00 \mathrm{~g}, 5.5 \mathrm{mmol}$ ), 1-乙基-(3-二甲基氨基丙 基)碳酰二亚胺盐酸盐(EDCI, $1.27 \mathrm{~g}, 6.6 \mathrm{mmol}$ ), 1-差基 苯并三唑(HOBT, $0.90 \mathrm{~g}, 6.6 \mathrm{mmol}$ )和 $N, N$-二异丙基乙胺 (DIPEA, $2.56 \mathrm{~g}, 20.0 \mathrm{mmol}$ )溶解在装有 $30 \mathrm{~mL} N, N$-二甲 基甲酰胺的圆底烧瓶中. 向该反应体系中加入 $(R / S)$-苯 乙胺 $(0.74 \mathrm{~g}, 6.1 \mathrm{mmol})$ 并持续搅拌 $12 \mathrm{~h}$, 通过 TLC 监测 反应过程. 反应结束后, 将反应液倒入 $100 \mathrm{~mL}$ 水中, 乙
酸乙酯 $(25 \mathrm{~mL} \times 3)$ 萃取, 用 $1 \mathrm{~mol} \cdot \mathrm{L}^{-1} \mathrm{HCl}$ 溶液 $(10 \mathrm{~mL} \times$ $3)$ 反复洗涤有机层. 随后, 用无水 $\mathrm{Na}_{2} \mathrm{SO}_{4}$ 对有机层进行 干燥, 减压蒸除乙酸乙酯, 得到黄色油状液体 3a. 在室 温条件下将 $\mathbf{3 a}$ 溶解在 $30 \mathrm{~mL} \mathrm{CH} \mathrm{mH}_{3} \mathrm{OH}$, 向该溶液中加 入 $\mathrm{NaOH}(0.44 \mathrm{~g}, 11.0 \mathrm{mmol})$ 并搅拌 $10 \mathrm{~h}$, 通过 TLC 监测 反应过程. 反应结束后, 用 $1 \mathrm{~mol} \cdot \mathrm{L}^{-1} \mathrm{HCl}$ 调节反应液 $\mathrm{pH}=4$, 体系中析出大量白色固体. 粗产品通过乙酸乙 酯重结晶得到白色晶体 $4 \mathrm{a}$, 产率为 $46 \%$.

\section{$4.45 a$ 和配体 $L^{R R}$ 和 $L^{S S}$ 的合成}

将 $4 \mathrm{a}(1.50 \mathrm{~g}, 5.6 \mathrm{mmol})$ 溶解在装有 $5 \mathrm{~mL} \mathrm{SOCl}_{2}$ 的 Schlenk 瓶中, 室温下搅拌 $6 \mathrm{~h}$, 并通过 TLC 监测反应过 程. 反应结束后, 蒸馏除去剩余的 $\mathrm{SOCl}_{2}$, 得到白色固 体. 将白色固体溶解在装有 $30 \mathrm{~mL}$ 无水 $\mathrm{CH}_{2} \mathrm{Cl}_{2}$ 的圆底 烧瓶中，向该体系中加入 4-二甲氨基吡啶(DMAP, 0.12 $\mathrm{g}, 1.0 \mathrm{mmol})$ 和三乙胺 $(0.48 \mathrm{~g}, 8.0 \mathrm{mmol})$. 随后, 缓慢滴 加乙二胺 $(0.12 \mathrm{~g}, 2.0 \mathrm{mmol})$ 的无水 $\mathrm{CH}_{2} \mathrm{Cl}_{2}$ 溶液, 搅拌 10 $\mathrm{h}$, 通过 TLC 监测反应过程. 反应结束后, 用 $1 \mathrm{~mol} \cdot \mathrm{L}^{-1}$ $\mathrm{HCl}$ 溶液 $(10 \mathrm{~mL} \times 3)$ 、饱和 $\mathrm{NaHCO}_{3}$ 溶液 $(10 \mathrm{~mL} \times 3)$ 和 水(10 mL)按顺序洗涤反应液. 洗涤后的有机层用无水 $\mathrm{Na}_{2} \mathrm{SO}_{4}$ 干燥, 蒸馏除去 $\mathrm{CH}_{2} \mathrm{Cl}_{2}$ 溶剂, 得到白色固体. 粗 产品在混合溶剂 $V\left(\mathrm{CH}_{2} \mathrm{Cl}_{2}\right): V(n$-hexane $)=1: 20$ 中重 结晶得到白色晶体 $\mathbf{L}^{R R / S S}(0.54 \mathrm{~g})$, 产率约为 $47 \%$.

\section{5 配合物 $\left[\mathrm{Ln}_{2}\left(\mathrm{~L}^{R R}\right)_{3}\right](\mathrm{OTf})_{6}$ 和 $\left[\mathrm{Ln}_{2}\left(\mathrm{~L}^{S S}\right)_{3}\right](\mathrm{OTf})_{6}$ 的合 成}

将 $\mathbf{L}^{R R / S S}(0.10 \mathrm{~g}, 0.18 \mathrm{mmol})$ 溶解在装有 $10 \mathrm{~mL}$ 无水 $\mathrm{CH}_{3} \mathrm{CN}$ 的圆底烧瓶中, 向该溶液中缓慢滴加 $\operatorname{Ln}(\mathrm{OTf})_{3}$ (0.12 mmol) 的无水 $\mathrm{CH}_{3} \mathrm{CN}$ 溶液, 搅拌 $24 \mathrm{~h}$. 反应结束 后, 减压蒸馏除去 $\mathrm{CH}_{3} \mathrm{CN}$, 得到粗产品 $(0.12 \mathrm{~g})$, 产率约 为 $71 \%$. 粗产品在混合溶剂 $V\left(\mathrm{CH}_{3} \mathrm{CN}\right): V\left(\mathrm{CH}_{2} \mathrm{Cl}_{2}\right)=$ $1: 10$ 的条件下缓慢析出, 得到晶态目标产物.

\section{References}

[1] (a) Lim, D.-Y. J. Korean Phys. Soc. 2006, 49, S505. (b) Werner, E. J.; Datta, A.; Jocher, C. J.; Raymond, K. N. Angew. Chem., Int. Ed. 2008, 47, 8568. (c) Huo, S.; Duan, P.; Jiao, T.; Peng, Q.; Liu, M. Angew. Chem., Int. Ed. 2017, 56, 12174. (d) Zinna, F.; Giovanella, U.; Di Bari, L. Adv. Mater. 2015, 27, 1791. (e) Victor, T. W.; O'Toole, K. H.; Easthon, L. M.; Ge, M.; Smith, R. J.; Huang, X.; Yan, H.; Chu, Y. S.; Chen, S.; Gursoy, D.; Ralle, M.; Imperiali, B.; Allen, K. N.; Miller, L. M. J. Am. Chem. Soc. 2020, 142, 2145.

[2] Huck, N. P. M.; Jager, W. F.; de Lange, B.; Feringa, B. L. Science 1996, 273, 1686.

[3] (a) Carr, R.; Evans, N. H.; Parker, D. Chem. Soc. Rev. 2012, 41 , 7673. (b) Shuvaev, S.; Fox, M. A.; Parker, D. Angew. Chem., Int. Ed. 2018, 57, 7488. (c) Shuvaev, S.; Suturina, E. A.; Mason, K; Parker, 
D. Chem. Sci. 2018, 9, 2996. (d) Staszak, K.; Wieszczycka, K.; Marturano, V.; Tylkowski, B. Coord. Chem. Rev. 2019, 397, 76.

[4] (a) Zinna, F.; Di Bari, L. Chirality 2015, 27, 1. (b) Shuvaev, S.; Starck, M.; Parker, D. Chem. - Eur. J. 2017, 23, 9974. (c) Yang, Y.; da Costa, R. C.; Fuchter, M. J.; Campbell, A. J. Nat. Photonics 2013, 7, 634. (d) Sethy, R.; Kumar, J.; Métivier, R.; Louis, M.; Nakatani, K.; Mecheri, N. M. T.; Subhakumari, A.; Thomas, K. G.; Kawai, T.; Nakashima, T. Angew. Chem., Int. Ed. 2017, 56, 15053. (e) Staszak, K.; Wieszczycka, K.; Marturano, V.; Tylkowski, B. Coord. Chem. Rev. 2019, 397, 76. (f) Shuvaev, S.; Suturina, E. A.; Mason, K.; Parker, D. Chem. Sci. 2018, 9, 2996.

[5] (a) Zhou, Y.; Li, H.; Zhu, T.; Gao, T.; Yan, P. J. Am. Chem. Soc. 2019, 141, 19634. (b) Liu, D.; Zhou, Y.; Zhang, Y.; Li, H.; Chen, P.; Sun, W.; Gao, T.; Yan, P. Inorg. Chem. 2018, 57, 8332. (c) Sugimoto, M.; Liu, X.-L.; Tsunega, S.; Nakajima, E.; Abe, S.; Nakashima, T.; Kawai, T.; Jin, R.-H. Chem. - Eur. J. 2018, 24, 6519. (d) Tan, Y. B; Okayasu, Y.; Katao, S.; Nishikawa, Y.; Asanoma, F.; Yamada, M.; Yuasa, J.; Kawai, T. J. Am. Chem. Soc. 2020, 142, 17653. (e) Deng, M.; Schley, N. D.; Ung, G. Chem. Commun. 2020, 56, 14813. (f) Hasegawa, Y.; Miura, Y.; Kitagawa, Y.; Wada, S.; Nakanishi, T.; Fushimi, K.; Seki, T.; Ito, H.; Iwasa, T.; Taketsugu, T.; Gon, M.; Tanaka, K.; Chujo, Y.; Hattori, S.; Karasawa, M.; Ishii, K. Chem. Commun. 2018, 54, 10695. (g) Zhang, J.; Dai, L.; Webster, A. M.; Chan, W. T. K.; Mackenzie, L. E.; Pal, R.; Cobb, S. L.; Law, G.-L. Angew. Chem., Int. Ed. 2021, 60, 1004.

[6] (a) Li, M.; Lin, W. B.; Fang, L.; Chen, C. F. Acta Chim. Sinica 2017, 75,1150 (in Chinese). (李猛, 林伟涁, 房蕾, 陈传峰, 化学学报, 2017, 75, 1150.) (b) Zhang, L.; Zhao, W. L.; Li, M.; Lu, H. Y.; Chen, C. F. Acta Chim. Sinica 2020, 78, 1030 (in Chinese). (张亮, 赵文 龙, 李猛, 吕海燕, 陈传峰, 化学学报, 2020, 78, 1030.) (c) Sun, Z.-B.; Liu, J.-K.; Yuan, D.-F.; Zhao, Z.-H.; Zhu, X.-Z.; Liu, D.-H.; Peng, Q.; Zhao, C.-H. Angew. Chem., Int. Ed. 2019, 58, 4840. (d) Jiang, Q.; Xu, X.; Yin, P.-A.; Ma, K.; Zhen, Y.; Duan, P.; Peng, Q.; Chen, W.-Q.; Ding, B. J. Am. Chem. Soc. 2019, 141, 9490. (e) Takaishi, K.; Yasui, M.; Ema, T. J. Am. Chem. Soc. 2018, 140, 5334. (f) Feuillastre, S.; Pauton, M.; Gao, L.; Desmarchelier, A.; Riives, A. J.; Prim, D.; Tondelier, D.; Geffroy, B.; Muller, G.; Clavier, G.; Pieters, G. J. Am. Chem. Soc. 2016, 138, 3990. (g) Cruz, C. M.; Castro-Fernández, S.; Maçôas, E.; Cuerva, J. M.; Campaña, A. G. Angew. Chem., Int. Ed. 2018, 57, 14782. (h) Liu, J. G.; Yin, F.; Hu, J.; Ju, Y. Chin. J. Org. Chem. 2021, 41, 1031. (in Chinese). (刘金果, 殷凤，胡君，巨勇，有机化学, 2021, 41, 1031.)

[7] (a) San Jose, B. A.; Matsushita, S.; Akagi, K. J. Am. Chem. Soc. 2012, 134, 19795. (b) San Jose, B. A.; Matsushita, S.; Akagi, K. J. Am. Chem. Soc. 2012, 134, 19795. (c) San Jose, B. A.; Yan, J.; Akagi, K. Angew. Chem., Int. Ed. 2014, 53, 10641. (d) Lee, S.; Kim, K. Y.; Jung, S. H.; Lee, J. H.; Yamada, M.; Sethy, R.; Kawai, T.; Jung, J. H. Angew. Chem., Int. Ed. 2019, 58, 18878.

[8] (a) Yang, D.; Duan, P.; Zhang, L.; Liu, M. Nat. Commun. 2017, 8, 15727. (b) Jiang, H.; Jiang, Y.; Han, J.; Zhang, L.; Liu, M. Angew. Chem., Int. Ed. 2019, 58, 785. (c) Hellou, N.; Srebro-Hooper, M.; Favereau, L.; Zinna, F.; Caytan, E.; Toupet, L.; Dorcet, V.; Jean, M.; Vanthuyne, N.; Williams, J. A. G.; Di Bari, L.; Autschbach, J.; Crassous, J. Angew. Chem., Int. Ed. 2017, 56, 823. (d) San Jose, B. A.; Matsushita, S.; Akagi, K. J. Am. Chem. Soc. 2012, 134, 19795. (e) Han, D.; Han, J.; Huo, S.; Qu, Z.; Jiao, T.; Liu, M.; Duan, P. Chem. Commun. 2018, 54, 5630. (f) Aoki, R.; Toyoda, R.; Kögel, J. F.; Sakamoto, R.; Kumar, J.; Kitagawa, Y.; Harano, K.; Kawai T.; Nishihara, H. J. Am. Chem. Soc. 2017, 139, 16024. (g) Reiné, P.; Ortuño, A. M.; Resa, S.; Álvarez de Cienfuegos, L.; Blanco, V.; Ruedas-Rama, M. J.; Mazzeo, G.; Abbate, S.; Lucotti, A.; Tommasini, M.; Guisán-Ceinos, S.; Ribagorda, M.; Campaña, A. G.; Mota, A.; Longhi, G.; Miguel, D.; Cuerva, J. M. Chem. Commun. 2018, $54,13985$.

[9] (a) Jiménez, J.-R.; Doistau, B.; Cruz, C. M.; Besnard, C.; Cuerva, J. M.; Campaña, A. G.; Piguet, C. J. Am. Chem. Soc. 2019, 141, 13244. (b) Aoki, R.; Toyoda, R.; Kögel, J. F.; Sakamoto, R.; Kumar, J.; Kitagawa, Y.; Harano, K.; Kawai, T.; Nishihara, H. J. Am. Chem. Soc. 2017, 139, 16024. (c) Hellou, N.; Srebro-Hooper, M.; Favereau, L.; Zinna, F.; Caytan, E.; Toupet, L.; Dorcet, V.; Jean, M.; Vanthuyne, N.; Williams, J. A. G.; Di Bari, L.; Autschbach, J.; Crassous, J. Angew. Chem., Int. Ed. 2017, 56, 8236.

[10] Lunkley, J. L.; Shirotani, D.; Yamanari, K.; Kaizaki, S.; Muller, G. J. Am. Chem. Soc. 2008, 130, 13814.
[11] Yeung, C.-T.; Chan, W. T. K.; Yan, S.-C.; Yu, K.-L.; Yim, K.-H.; Wong, W.-T.; Law, G.-L. Chem. Commun. 2015, 51, 592.

[12] Li, X.-Z.; Zhou, L.-P.; Yan, L.-L.; Yuan, D.-Q.; Lin, C.-S.; Sun, Q.-F. J. Am. Chem. Soc. 2017, 139, 8237.

[13] (a) Stomeo, F.; Lincheneau, C.; Leonard, J. P.; O'Brien, J. E.; Peacock, R. D.; McCoy, C. P.; Gunnlaugsson, T. J. Am. Chem. Soc. 2009, 131, 9636. (b) Barry, D. E.; Kitchen, J. A.; Pandurangan, K. Savyasachi, A. J.; Peacock, R. D.; Gunnlaugsson T. Inorg. Chem. 2020, 59, 2646. (c) Comby, S.; Stomeo, F.; McCoy, C. P.; Gunnlaugsson, T. Helv. Chim. Acta 2009, 92, 2461. (d) Kotova, O.; Comby, S.; Pandurangan, K.; Stomeo, F.; O’Brien, J. E.; Feeney, M.; Peacock, R. D.; McCoy, C. P.; Gunnlaugsson, T. Dalton Trans. 2018, 47, 12308. (e) Lincheneau, C.; Peacock, R. D.; Gunnlaugsson, T. Chem. Asian J. 2010, 5, 500 .

[14] Yeung, C.-T.; Yim, K.-H.; Wong, H.-Y.; Pal, R.; Lo, W.-S.; Yan, S.-C.; Wong, M. Y.-M.; Yufit, D.; Smiles, D. E.; McCormick, L. J.; Teat, S. J.; Shuh, D. K.; Wong, W.-T.; Law, G.-L. Nat. Commun. 2017, 8, 1128.

[15] Zhang, T.; Zhang, G.-L.; Zhou, L.-P.; Guo, X.-Q.; Sun, Q.-F. Tetrahedron: Asymmetry 2017, 28, 550.

[16] (a) Zhou, Y.; Yao, Y.; Cheng, Z.; Gao, T.; Li, H.; Yan, P. Inorg. Chem. 2020, 59, 12850. (b) Han, G.; Zhou, Y.; Yao, Y.; Cheng, Z.; Gao, T.; Li, H.; Yan, P. Dalton Trans. 2020, 49, 3312.

[17] (a) Zhang, J.; Zhou, Y.; Yao, Y.; Cheng, Z.; Gao, T.; Li, H.; Yan, P. J. Mater. Chem. C 2020, 8, 6788. (b) Chen, A. Y.; Thomas, P. W.; Stewart, A. C.; Bergstrom, A.; Cheng, Z.; Miller, C.; Bethel, C. R.; Marshall, S. H.; Credille, C. V.; Riley, C. L.; Page, R. C.; Bonomo, R. A.; Crowder, M. W.; Tierney, D. L.; Fast, W.; Cohen, S. M. J. Med. Chem. 2017, 60, 7267. (c) Yan, L.-L.; Tan, C.-H.; Zhang, G.-L.; Zhou, L.-P.; Bünzli, J.-C.; Sun, Q.-F. J. Am. Chem. Soc. 2015, 137, 8550. (d) Li, X.-Z.; Zhou, L.-P.; Yan, L.-L.; Yuan, D.-Q.; Lin, C.-S.; Sun, Q.-F. J. Am. Chem. Soc. 2017, 139, 8237.

[18] Dutra, J. D. L.; Bispo, T. D.; Freire, R. O. J. Comput. Chem. 2014 35,772 .

[19] Telfer, S. G.; McLean, T. M.; Waterland, M. R. Dalton Trans. 2011, $40,3097$.

[20] Muller, G. In Luminescence of Lanthanide Ions in Coordination Compounds and Nanomaterials, Ed.: de Bettencourt-Dias, A., Wiley, Hoboken, 2014, pp. 77 124 .

[21] (a) Bonsall, S. D.; Houcheime, M.; Straus, D. A.; Muller, G. Chem Commun. 2007, 35, 3676. (b) Petoud, S.; Muller, G.; Moore, E. G.; Xu, J.; Sokolnicki, J.; Riehl, J. P.; Le, U. N.; Cohen, S. M.; Raymond, K. N. J. Am. Chem. Soc. 2007, 129, 77. (c) Seitz, M.; Do, K.; Ingram, A. J.; Moore, E. G.; Muller, G.; Raymond, K. N. Inorg. Chem. 2009, 48, 8469. (d) Leonard, J. P.; Jensen, P.; McCabe, T; O’Brien, J. E.; Peacock, R. D.; Kruger, P. E.; Gunnlaugsson, T. J. Am. Chem. Soc. 2007, 129, 10986. (e) Shi, N.; Wang, R.; Wang, X.; Tan, J.; Guan, Y.; Li, Z.; Wan, X.; Zhang, J. Chem. Commun. 2019 , 55, 1136. (f) Yeung, C.-T.; Yim, K.-H.; Wong, H.-Y.; Pal, R.; Lo, W.-S.; Yan, S.-C.; Yee-Man Wong, M.; Yufit, D.; Smiles, D. E.; McCormick, L. J.; Teat, S. J.; Shuh, D. K.; Wong, W.-T. Law, G.-L. Nat. Commun. 2017, 8, 1128. (g) Górecki, M.; Carpita, L.; Arrico, L.; Zinna, F.; Di Bari, L. Dalton Trans. 2018, 47, 7166. (h) Kotova, O.; Blasco, S.; Twamley, B.; O’ Brien, J.; Peacock, R. D.; Kitchen, J. A.; Martínez-Calvo, M.; Gunnlaugsson, T. Chem. Sci. 2015, 6, 457. (i) Okutani, K.; Nozaki, K.; Iwamura, M. Inorg. Chem. 2014, 53, 5527. (j) Leonzio, M.; Melchior, A.; Faura, G.; Tolazzi, M.; Zinna, F.; Di Bari, L.; Piccinelli, F. Inorg. Chem. 2017, 56, 4413.

[22] (a) Hua, K. T.; Xu, J.; Quiroz, E. E.; Lopez, S.; Ingram, A. J.; Johnson, V. A.; Tisch, A. R.; de Bettencourt-Dias, A.; Straus, D. A.; Muller, G. Inorg. Chem. 2012, 51, 647. (b) Lunkley, J. L.; Shirotani, D.; Yamanari, K.; Kaizaki, S.; Muller, G. Inorg. Chem. 2011, 50, 12724 .

[23] (a) Miyata, K.; Nakagawa, T.; Kawakami, R.; Kita, Y.; Sugimoto, K.; Nakashima, T.; Harada, T.; Kawaiand T.; Hasegawa, Y. Chem. Eur. J. 2011, 17, 521. (b) Hasegawa, Y.; Ohkubo, T.; Nakanishi, T.; Kobayashi, A.; Kato, M.; Seki, T.; Ito, H.; Fushimi, K. Eur. J. Inorg. Chem. 2013, 5911. (c) Zhu, T.; Chen, P.; Li, H.; Sun, W.; Gao, T.; Yan, P. Phys. Chem. Chem. Phys. 2015, 17, 16136.

[24] (a) Latva, M.; Takalo, H.; Mukkala, V.-M.; Matachescu, C.; Rodriguez-Ubis, J. C.; Kankare, J. J. Lumin. 1997, 75, 149. (b) Steemers, F. J.; Verboom, W.; Reinhoudt, D. N.; van der Tol, E. B.; Verhoeven, J. W. J. Am. Chem. Soc. 1995, 117, 9408. 